\title{
Editorial
}

\section{Experimental Tests of Quantum Gravity and Exotic Quantum Field Theory Effects}

\author{
Emil T. Akhmedov, ${ }^{1,2,3}$ Stephen Minter, ${ }^{4}$ Piero Nicolini, ${ }^{5,6}$ and Douglas Singleton ${ }^{7}$ \\ ${ }^{1}$ Moscow Institute of Physics and Technology, Institutskii per. 9, Dolgoprudny, Moscow 141700, Russia \\ ${ }^{2}$ International Laboratory of Representation Theory and Mathematical Physics, National Research University Higher School of \\ Economics and Moscow Institute of Physics and Technology, Institutskii per. 9, Dolgoprudny 141700, Russia \\ ${ }^{3}$ Institute for Theoretical and Experimental Physics, B. Cheremushkinskaya, No. 25, Moscow 117218, Russia \\ ${ }^{4}$ Vienna Center for Quantum Science and Technology, University of Vienna, Boltzmanngaße 5, 1090 Vienna, Austria \\ ${ }^{5}$ Frankfurt Institute for Advanced Studies (FIAS), Ruth-Moufang-Straße 1, 60438 Frankfurt am Main, Germany \\ ${ }^{6}$ Institut für Theoretische Physik, Johann Wolfgang Goethe-Universität, Max-von-Laue-Straße 1, 60438 Frankfurt am Main, Germany \\ ${ }^{7}$ Physics Department, California State University, Fresno, Fresno, CA 93740, USA
}

Correspondence should be addressed to Douglas Singleton; dougs@csufresno.edu

Received 9 June 2014; Accepted 9 June 2014; Published 23 June 2014

Copyright (C) 2014 Emil T. Akhmedov et al. This is an open access article distributed under the Creative Commons Attribution License, which permits unrestricted use, distribution, and reproduction in any medium, provided the original work is properly cited. The publication of this article was funded by $\mathrm{SCOAP}^{3}$.

Physics at its core is an experimental pursuit. If one theory does not agree with experimental results, then the theory is wrong. However, it is becoming harder and harder to directly test some theories of fundamental physics at the high energy/small distance frontier exactly because this frontier is becoming technologically harder to reach. The Large Hadron Collider is getting near the limit of what we can do with present accelerator technology in terms of directly reaching the energy frontier. The motivation for this special issue was to try and collect together ideas and potential approaches to experimentally probe some of our ideas about physics at the high energy/small distance frontier. Some of the papers in this special issue directly deal with the issue of what happens to spacetime at small distance scales. In the paper by A. Aurilia and E. Spallucci a picture of quantum spacetime is given based on the effects of ultrahigh velocity length contractions on the structure of the spacetime. The work of P. Nicolini et al. further pursues the idea that spacetime has a minimal length. The consequences of this minimal length are investigated in terms of the effects it would have on the gravitational collapse of a star to form a black hole. In the article by G. Amelino-Camelia et al. the quantum structure of spacetime is studied through the Fermi LAT data on the Gamma Ray Burst
GRB130427A. The article by S. Hossenfelder addressed the question of whether spacetime is fundamentally continuous or discrete and postulates that in the case when spacetime is discrete it might have defects which would have important observational consequences.

Shortly after the formulation of general relativity Theodor Kaluza proposed the idea that one could unify gravity and electromagnetism by allowing spacetime to have four spatial dimensions rather than the three spatial dimensions which are normally observed. Depending on the size of the extra dimensions, one might have interesting and observable consequences connected with these extra dimensions. The article by M. Gogberashvili and P. Midodashvili investigates gravitational and scalar field standing waves in a five-dimensional spacetime (one time dimension and four spatial dimensions). Their model gives rise to standing wave modes which could lead to observable consequences at accelerators. The paper by A. Chopovsky et al. argues that there are constraints in certain extra dimensional models which lead to an absence of Kaluza-Klein excitations.

In the past decade or more, satellite experiments such as COBE, WMAP, and PLACNK have given us new insight into the fundamental workings of the Universe. The results 
of these and other observational cosmological experiments have shown that there is much in the Cosmos that we still do not understand (e.g., dark energy), but through studying these unknown quantities hopefully a better understanding of the fundamental laws of physics will emerge. The work of R. Peron studies and discusses how the LAGEOS satellite, launched mainly for geodetic and geodynamical purposes, is being used to perform studies of fundamental physics. In the paper by Y. V. Dumin the application of nonlocal quantum effects to the large scale structure of the Universe is investigated, using an analogy with superconducting multiJosephson-junction loops and ultracold gases in periodic potentials. The work of T. Asselmeyer-Maluga and J. Król uses the idea of exotic smoothness of spacetime to propose that the initial inflationary phase of the Universe was caused by a topological phase transition.

One of the first steps in the direction of formulating a quantum theory of gravity is Stephen Hawking's results that black holes give off a thermal radiation at a temperature inversely proportional to the mass of the black hole. This result is the consequence of studying quantum fields in a curved spacetime background. However, astrophysical black holes have Hawking temperatures too small to detect with present technology. To this end people have looked at analog systems which exhibit phenomenon similar to Hawking radiation. The work of P. Castorina and $\mathrm{H}$. Satz looks at the idea that some unusual features of high energy collision can be explained by invoking thermal hadron production via Hawking-Unruh-like radiation. The paper by R. Balbinot and A. Fabbri looks at the Hawking effect in terms of the analog system of Bose-Einstein condensates, where one has acoustic black holes, with quanta of light (i.e., photons) replaced by quanta of sound (i.e., phonons) and with the black hole event horizon replaced by an acoustic horizon.

Much of modern physics is based on symmetries (e.g., gauge symmetries and spacetime symmetries) and in some cases the breaking of the symmetry (e.g., the breaking of some of the gauge symmetries of the Standard Model of particle physics via the Higgs mechanism). The paper by S. A. Hojman and B. Koch looks at the gauge symmetry of E\&M which would, if unbroken, predict that the photon is exactly massless. S. A. Hojman and B. Koch derive an astrophysical lower bound on the photon mass, which when combined with the present experimental upper bound would imply that the photon is required to be exactly massless-the gauge symmetry of E\&M is not broken. The work by A. G. Lebed studies potential violations of the Equivalence Principle, which forms the conceptual basis of general relativity via the superposition of quantum states. The violations of the Equivalence Principle proposed by A. G. Lebed lead to experiments which can be performed using satellites in Earth orbit.

By editing the present volume, we offer a panoramic overview of the experimental and phenomenological aspects of quantum gravity and exotic quantumfield theory effects to inspire future investigation in this fundamental and developing field.

Emil T. Akhmedov Stephen Minter Piero Nicolini

Douglas Singleton 

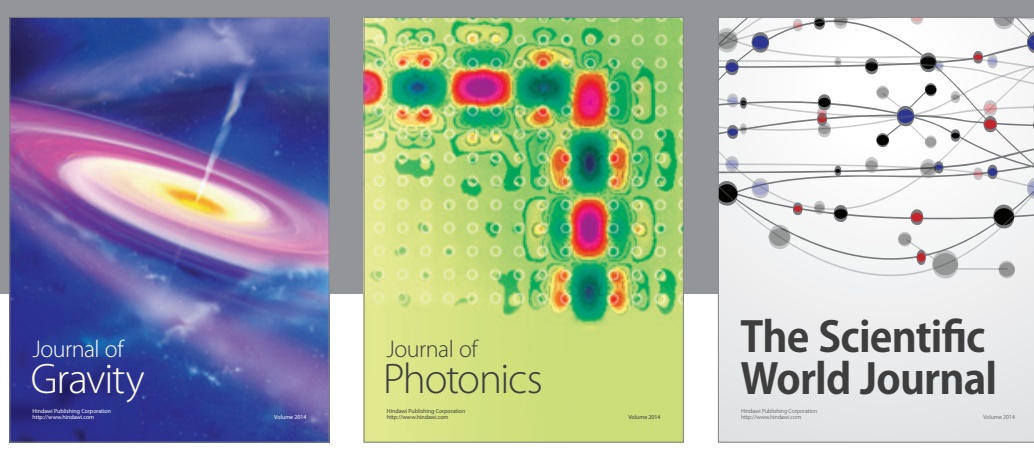

The Scientific World Journal
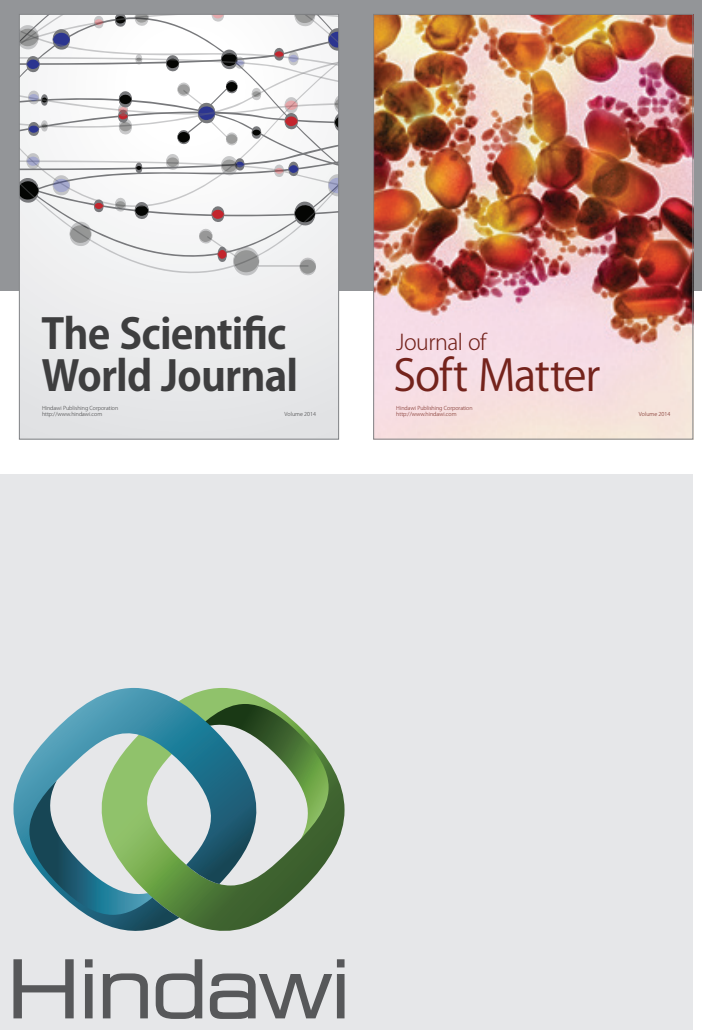

Submit your manuscripts at

http://www.hindawi.com

nternational Journal of

Statistical Mechanics
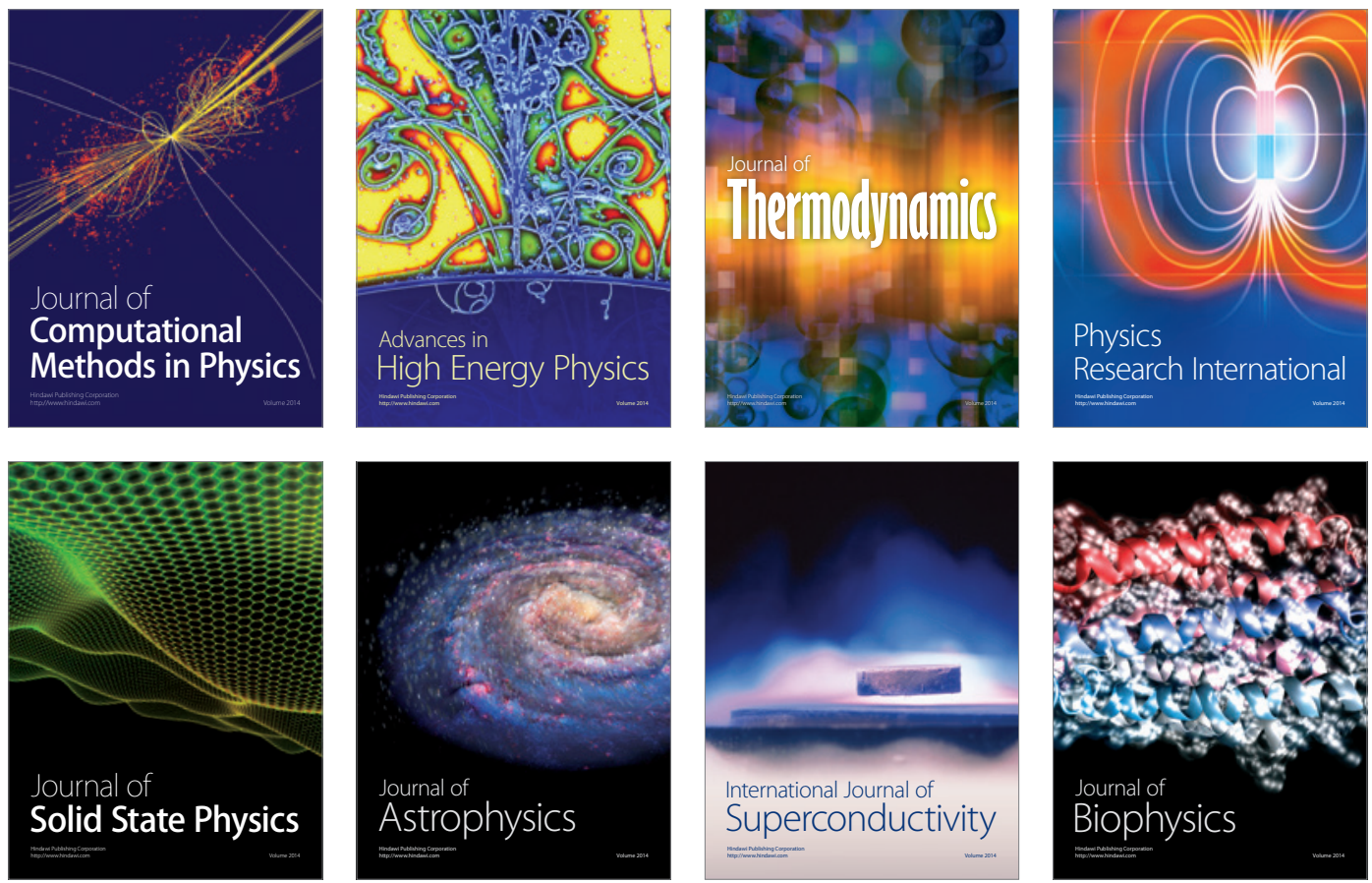
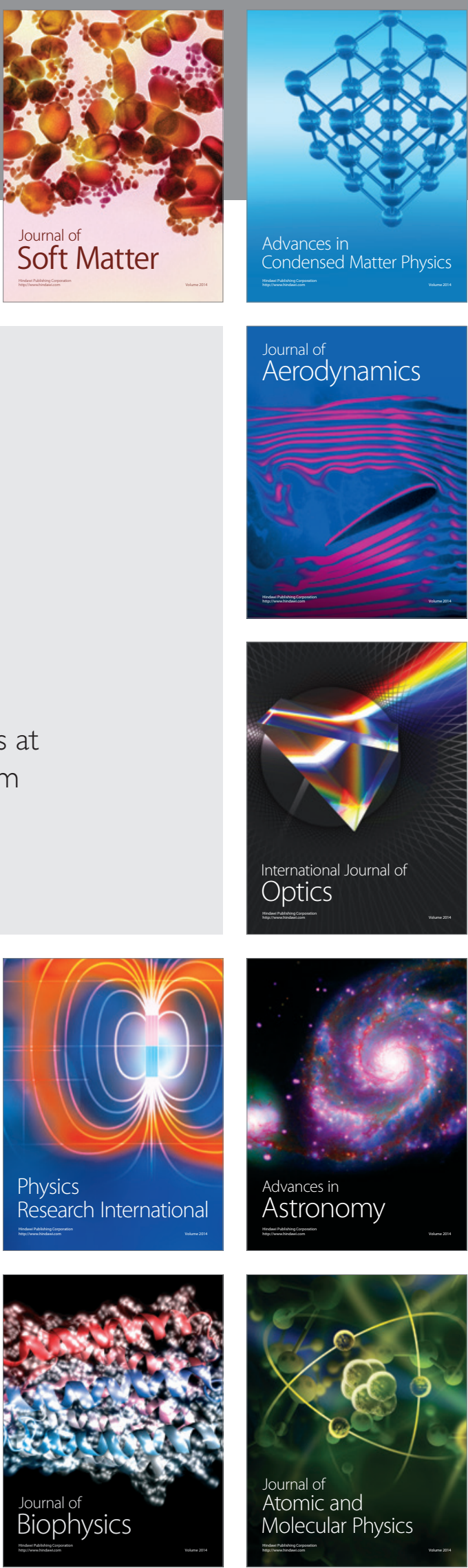